\title{
On the capacity of a SIMO land mobile satellite system at C-band: polarized and depolarized received field
}

\author{
Nektarios Moraitis ${ }^{1 *}$, Péter Horváth ${ }^{2}$, Philip Constantinou ${ }^{1}$ and István Frigyes ${ }^{2}$
}

\begin{abstract}
Land mobile satellite can exploit multiple input multiple output techniques to achieve high transmission rates. This article evaluates, theoretically, the capacity of the single input multiple output system utilizing uniform linear arrays at the receiver terminal for satellite applications. The theoretical study is performed at C-band and accounts for different shadowing conditions. Additionally, polarization effects are introduced and capacity results are presented that take into account the depolarization. For this investigation, a model for the scattering caused depolarization based on Stokes parameters is applied. Decrease of channel capacity is determined for some special cases both for Rayleigh fading and for the ULA with different number of receive antennas.
\end{abstract}

Keywords: SIMO satellite, Channel model, Downlink capacity, Uniform linear arrays, Depolarization modeling, Depolarized field

\section{Introduction}

Multiple antenna wireless systems, and particularly multiple input multiple output (MIMO) systems, yield unprecedented possibilities of innovation in wireless communications. While in principle MIMO advantages are achievable both with free space channels (e.g. [1,2]) and multipath channels, practical reasons prefer the latter. As a consequence satellite links are not well suited for MIMO applications: the path-length is extremely long, propagation along most of the path is free space and antennas are-nearly always - of very narrow beam. Furthermore, it is shown in the literature that the other forms of diversity (mainly satellite diversity, where two satellites orbiting far from each other serve as diversity terminals) cause severe intersymbol interference and raise synchronization issues [2]. The solution of these problems is not simple at all and details are not yet clear. Possibilities of polarization diversity, on the other hand, are more restricted than those of, e.g. space or frequency diversity. Having taken this into account, it seems reasonable to investigate what advantages (if any)

\footnotetext{
* Correspondence: morai@mobile.ntua.gr

${ }^{1}$ Institute of Communications and Computer Systems, National Technical University of Athens, Athens, Greece

Full list of author information is available at the end of the article
}

of a true MIMO system can be achieved with architectures appropriate in satellite systems, these being more conservative than MIMO architecture, i.e. single input multiple output (SIMO) in the downlink. In particular, if channel capacity can significantly be increased by the application of multi-antenna satellite systems. The problem is related to MIMO studies as the question itself and concepts and methods applied have existed since the advent of MIMO in the mid 1990s. There are few articles dealing with the MIMO satellite topic. For example, King et al. [3] give a physical-statistical model and compute the capacity of a $2 \times 2$ MIMO system. Further articles involved with MIMO satellite measurements are [4-6], whereas [7] investigates the modelling of the satellite MIMO channel emphasizing on polarization.

The aim of this article is to achieve a step on this path. A satellite downlink is investigated and our goal is to determine the channel capacity. The investigated system is SIMO, i.e. there is one transmit antenna onboard the satellite and a vertically polarized uniform linear array (ULA) receive antenna at the receiver terminal. Although it is known from theory that this structure yields only logarithmic increase of capacity versus the element number of the antenna, significant shift in signal-to- 
noise ratio (SNR) can be possible with appropriate environment and design. The number of applications using global navigation satellite system positioning is increasing steadily and currently the European Space Agency explores the possibility of satellite navigation signals operating in an already allocated frequency band for satellite radio navigation around $5 \mathrm{GHz}$ [8]. For that reason, the study is performed at C-band $(5.2 \mathrm{GHz})$, for a light and heavy shadowed environment.

Depolarization can change channel characteristics, including capacity (usually neglected in single-polarized situations). Therefore, the second step is to examine the channel capacity introducing SIMO depolarization scenarios and compare the difference with the polarized state. The problem of depolarization is investigated in a more general framework. In that, usual channel models-statistical like Rayleigh, Rice, Corazza-Vatalaro, etc., or physical, like ray tracing, full-wave electromagnetic models (or that used in this article for polarized SIMO) - are regarded as conditional models based on the loss due to polarization mismatch of the receive antenna. In order to determine statistics of the condition, a model described in [9], based on Stokes-parameters, is proposed. To give some general insight into the role of depolarization the model is also applied to various single input single output (SISO) and SIMO Rayleigh-fading situations. In several cases closed-form results were obtained and verified by simulation providing ergodic capacity results.
The remainder of the article is organized as follows. In Section 2 we describe the propagation scenario and geometry, the channel model and the capacity calculation methodology. Section 3 presents the outage capacity results for the polarized state of the channel. In Section 4 , unconditional statistics of representative channel models with representative depolarization models are determined, and, based on that, ergodic capacity of some SISO and SIMO situations is calculated. Finally, Section 5 is devoted to conclusions summarizing this study.

\section{Capacity evaluation methodology Propagation scenario}

The propagation scenario utilized to evaluate the channel capacity is illustrated in Figure 1. We assume that the direct component arrives at the mobile terminal having an angle-of-arrival (AoA), $\theta_{0}$, in the vertical plane of propagation. The multipath components arrive at the receiver antenna elements according to the angular distribution of scatterers as depicted, three-dimensionally, in Figure 1 . The mobile receiver is moving along the $x$-axis as indicated, heading away from the satellite. The multipath components are uniformly distributed within a sector with angular spread $\alpha$, where $\alpha$ may vary between 0 and $2 \pi$. This circle of scatterers has a radius of $S_{R}$ as shown in Figure 1. Since the receiver is moving away from the satellite, according to the proposed propagation scenario, the relationship between the angle of the direct component and the elevation angle of the satellite is

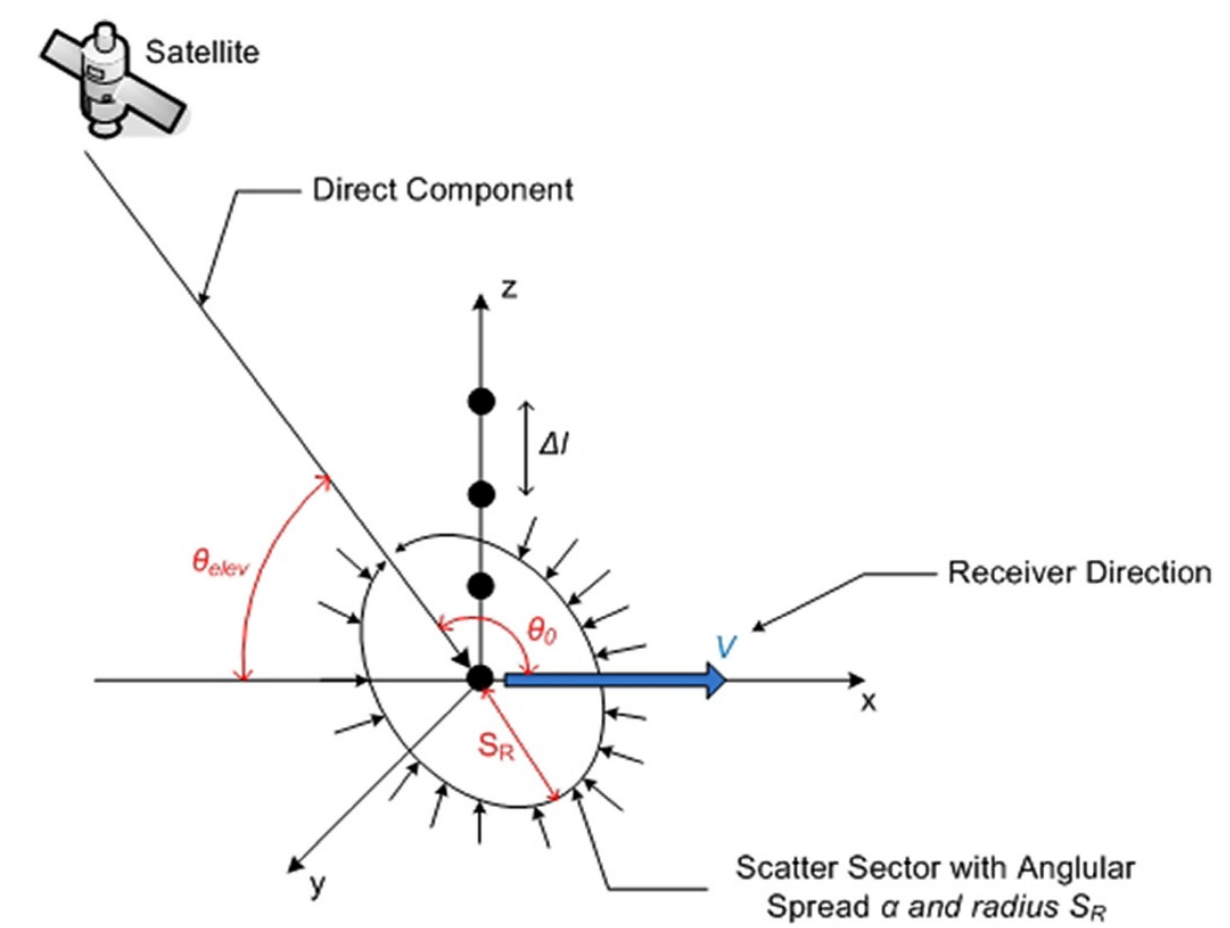

Figure 1 SIMO satellite propagation scenario, and distribution of multipath. 
$\theta_{0}=\pi-\theta_{\text {elev }}$. Additionally, the number of the scatterers depends on the angular spread and it is given from $L=50 a / \pi$, where $\alpha$ is between 0 and $2 \pi$.

The time-varying (since we have a mobile terminal) received complex envelope can be described by the following relationship:

$$
\begin{aligned}
\tilde{b}(t, \theta) & =\tilde{P}_{0}(t) \sqrt{\frac{K}{K+1}} \delta\left(\theta-\theta_{0}\right) \\
& +\frac{1}{\alpha} \sqrt{\frac{1}{K+1} \sum_{i=1}^{L}\left[\frac{\tilde{P}_{i}\left(t, \theta_{i}\right)}{\sum \tilde{P}_{i}}\right]}
\end{aligned}
$$

where $\tilde{b}$ is the time-varying angular-dependent complex envelope, $\theta_{O}$ is the angle at which the direct (line-ofsight) component arrives from the satellite to the mobile terminal, $\tilde{P}_{0}(t)$ is the time-varying received complex envelope of the direct component, $\tilde{P}_{i}\left(t, \theta_{i}\right)$ is the timevarying received complex envelope of each multipath component initiated by the $i^{\text {th }}$ scatterer at specific angle $\theta_{i}$ around the array, $L$ is the total number of the scatterers and $K$ is the ratio between the direct and the multipath components ( $K$-factor).

In order to produce the signal samples of the time-varying complex envelope of the direct and multipath components, respectively, we utilized the circuit implementation presented in Figure 2. The upper part stands for the direct component time-series calculation where the phase variations are introduced in the direct signal and then are multiplied with the produced lognormal samples. In the specific model, the constant phase increment $\Delta \phi=2 \pi f_{\max } \cos (\phi) \cos \left(\pi-\theta_{\text {elev }}\right) T_{s}$, with $\phi$ and $\theta_{\text {elev }}$ being the relative azimuth (route orientation with respect to the satellite) and elevation angles, respectively. Additionally, $T_{s}$ is the sampling interval in seconds used in the time-series synthesizer and $f_{\max }=V / \lambda$ is the maximum Doppler spread with $V$ being the mobile velocity in $\mathrm{m} / \mathrm{s}$ and $\lambda$ the signal wavelength in meters.

The lower part in Figure 2 is generating the timevarying complex envelope for each $i^{\text {th }}$ scatterer around the array (multipath components). The Rayleigh distributed series are spectrally shaped and multiplied by a slowly varying lognormal series thus modulating the mode of the Rayleigh series. The specific circuit implements the Suzuki distributed time-series [10]. Fast variations are ruled by Doppler spread mainly due to the terminal's motion. The Doppler spread is envisaged as a Butterworth filter being a more realistic approach for satellite cases. The Doppler filters would be narrower than when the overall multipath is simulated. Therefore, the Doppler spread for each one of the scatterers is $f_{i}=(V / \lambda) \cos \left(\theta_{i}\right) / L$ where $\theta_{i}$ is the angle between each $i^{\text {th }}$ scatterer and the direction of the receiver, whereas $L$ is the total number of the scatterers.

Taking into account a GEO satellite (fixed in the sky) we assume that the Doppler shift caused by the satellite motion is zero. For the rest of the simulation procedure,

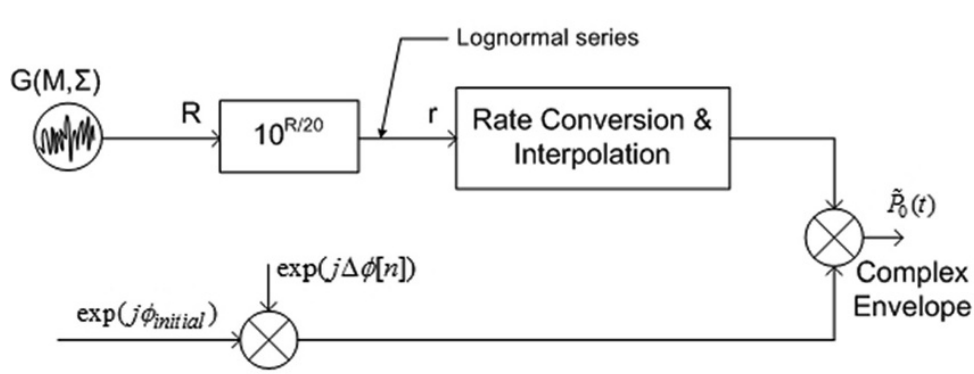

$\mathrm{G}(0,1)$

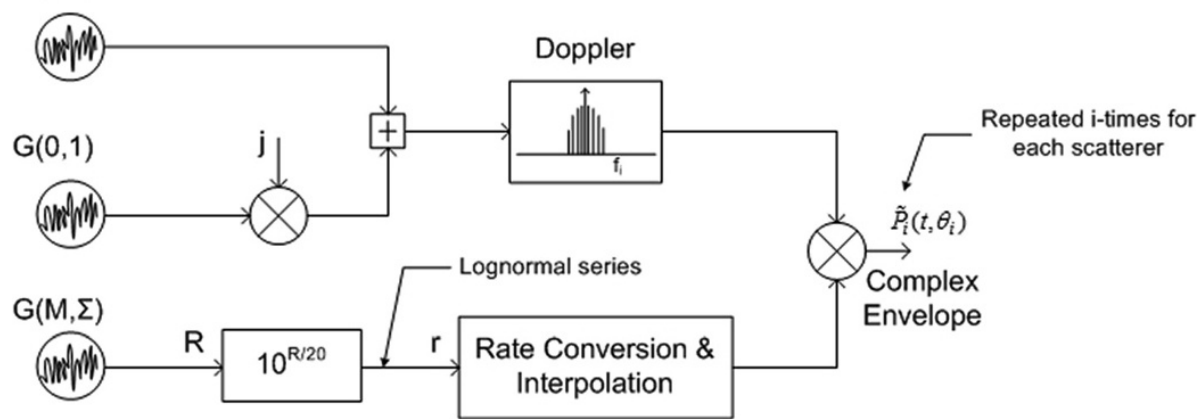

Figure 2 Circuit implementation of the time-varying received complex envelope of the direct component and the multipath components. 
we selected $\phi=0^{\circ}$ (since the mobile is moving along the $x$-axis as shown in Figure 1) with a mobile terminal speed of $30 \mathrm{~km} / \mathrm{h}$. The sampling frequency was chosen 16 times the Doppler spread each time. In the proposed model, in case of a light shadowing scenario, we select $M=1.13 \mathrm{~dB}, \Sigma=1 \mathrm{~dB}$ and $K=6 \mathrm{~dB}$, whereas in heavy shadowing scenario we select $M=-9.38 \mathrm{~dB}, \Sigma=2.5 \mathrm{~dB}$ and $K=-2.22 \mathrm{~dB}$, respectively [11].

In order to calculate the capacity of a SISO system with one antenna element at both terminals, it is necessary to calculate the channel response, $h(t)$, between the transmit and the receive antenna [12]. The time-varying channel impulse response of the complex angular profiles considering a narrowband system can be described by

$$
\tilde{h}(t, \theta)=\sum_{i=0}^{L} \tilde{P}_{i}(t) e^{j \phi_{i}} \delta\left(\theta-\theta_{i}\right)
$$

where $\tilde{P}_{i}(t)$ is the time-varying received complex envelope of each arrival $L$ at a specific angle $\theta_{i}$. The $e^{j \phi_{i}}$ term represents a statistically independent random phase associated with each arrival, where $\phi_{i}$ is uniform $[0,2 \pi)$. In case of a SIMO system, with one transmit and $M_{R}$ receive antenna elements, the complex channel vector $\tilde{\mathbf{h}}$ has to be calculated. In this study, we assumed ULA antennas at the receiver terminal where the elements are positioned along $z$-axis of propagation with $\Delta l$ spacing and horizontal polarization. Hence, for the specified antenna geometry the array response vector at the receiver is given by

$$
\mathbf{a}_{R}\left(\theta_{R}\right)=\left[1, e^{-j \frac{2 \pi}{\lambda} \Delta l \cos \theta_{R}}, \ldots, e^{\left.-j \frac{2 \pi}{\lambda} \Delta l\left(M_{R}-1\right) \cos \theta_{R}\right]^{T}}\right.
$$

where $\lambda$ is the wavelength, $\theta_{R}$ is the angle with respect to the antenna broadside of the impinging at the receiver array propagation path arriving at the receive antennas. The time-varying vector $\tilde{h} \in \mathrm{C}^{M_{R} \times 1}$ can be obtained by the matrix representation, which in case of a ULA configuration is given by the following equation

$$
\tilde{\mathbf{h}}(t)=\left[\begin{array}{lll}
\mathbf{a}\left(\theta_{R, 1}\right) & \ldots & \mathbf{a}\left(\theta_{R, L}\right)
\end{array}\right] \cdot \tilde{\mathbf{b}}(t)
$$

where $L$ is the number of propagation paths calculated by the vertical angular power profile at each position, $\mathbf{a}\left(\theta_{R, i}\right)$ is the array response vector for the $i^{\text {th }}$ path, and $\tilde{b} \in C^{L \times 1}$ is the vector representation of (1). Hence, from (1) if we know the AoA of each one of the $L$ propagation paths, the antenna elements and their spacing, we can calculate the time-varied complex channel vector $\tilde{\mathbf{h}}$. Finally, after calculating the channel vector $\tilde{\mathbf{h}}$ we can easily estimate the channel capacity as a function of the averaged SNR, assuming a channel unknown to the transmitter, according to the expression [12]

$$
C_{\mathrm{SIMO}}(t)=\log _{2}\left(1+\rho \sum_{i=1}^{M_{R}}\left|\tilde{h}_{i}(t)\right|^{2}\right)
$$

where $\rho$ is the averaged SNR at each receiver branch. The capacity is referred as the error free spectral efficiency, or the data rate per unit bandwidth that can be sustained reliably over the channel.

\section{Capacity evaluation procedure}

The steps in order to evaluate the capacity of the proposed SIMO satellite scenario are the following.

- Initially the frequency (in $\mathrm{GHz}$ ), the shadowing conditions (mean power $M$ and the standard deviation $\Sigma$ in $\mathrm{dB}$ ), the $K$-factor (in $\mathrm{dB}$ ), the velocity of the terminal (in $\mathrm{km} / \mathrm{h}$ ), the element spacing $\Delta l$ and the SNR are given.

- Then, the angular spread $a$ (in radians), as well as the scatter annulus with radius $S_{R}$ are determined. For example if $a=2 \pi$ then the number $L$ of the multipath components within the sector would be 100 .

- The elevation angle between the mobile terminal and the satellite is assigned and for each angle in the interval $\left[\theta_{0}-\pi, \theta_{0}+\pi\right]$ we calculate the time-varying complex envelope of the received signal for the direct as well as for each $i^{\text {th }}$ multipath component according to the model in Figure 2.

- According to the given elevation angle the direct component is multiplied by the factor $\sqrt{K /(K+1)}$, and each of the $i^{\text {th }}$ multipath components by the factor $\sqrt{1 /(K+1)}$ as shown by (1).

- Hence, a time-varying vector $\tilde{\mathbf{b}}(t)$ is created, which contains the received complex envelope as a function of angle of arrival, for each one of the $L$ propagation components (direct and multipath).

- Then, the steering vector of the receiver antenna is calculated according to (3), and from (4) the time-varying channel vector $\tilde{\mathbf{h}}(t)$ is extracted. The direct component is in position $\tilde{h}_{1}(t)$.

- Finally, we evaluate the outage channel capacity using (5), from which the cumulative distribution function (CDF) is extracted.

For the rest of the simulation procedure, we select the space element $\Delta l=\lambda$ so as to have a compact receiver antenna, and for the capacity calculation we have assume an SNR of $10 \mathrm{~dB}$. 


\section{Outage capacity results with polarized received field}

In this section, we present the results of the simulation procedure with polarization taken into account. Figure 3 presents the capacity CDF for heavy and light shadowing scenario at C-band $(5.2 \mathrm{GHz})$. Two or four elements have been considered at the receiver array as well as SISO case is presented for comparison. It is evident that the capacity improves with the number of the elements, in comparison with SISO case, as it is expected. Additionally, in the heavy shadowing scenario the capacity drops significantly comparable with the light shadowing case. Averaging the median value results $(50 \%$ in the $\mathrm{CDF})$, the capacity decreases about $1 \mathrm{~b} / \mathrm{s} / \mathrm{Hz}$. Overall, very sufficient capacities are achieved varying between 3.8 and $8.7 \mathrm{~b} / \mathrm{s} / \mathrm{Hz}$ (median values) in comparison with SISO case.

Figure 4 presents the average capacity of a SIMO system at $\mathrm{C}$-band with a variable angular spread, $a$, having either two or four elements at the receiver considering a light shadowing scenario $(K=6 \mathrm{~dB})$. The SNR is taken $10 \mathrm{~dB}$, the elevation angle $60^{\circ}$, and the scatterers' circle radius $50 \mathrm{~m}$. The angular spread takes on values between $30^{\circ}$ and $360^{\circ}$, in steps of $30^{\circ}$. The time-averaged capacity is given by

$$
\bar{C}_{\text {SIMO }}=\left\langle\log _{2}\left(1+\rho \sum_{i=1}^{M_{R}}\left|\tilde{h}_{i}(t)\right|^{2}\right)\right\rangle
$$

where $\langle\cdot\rangle$ denotes time average. The capacity increases as the angular spread increases since the multiple elements take advantage of the rich scattering environment.
We have to comment here that the spatial correlation between the antennas at the receive array depends strongly on the AoA, antenna spacing, angular spread and the azimuth power profile. A larger sector $a$ corresponds to the case of a richer scattering environment, therefore, a lower correlation between antennas. Thus, spatial correlation decreases as the angular spread is increased due to reduced correlation between the signals received at different antennas. This has the effect of providing better spatial diversity gains. The capacity is improved about $5 \mathrm{~b} / \mathrm{s} / \mathrm{Hz}$, in average, if angle $a$ increases from $30^{\circ}$ to $360^{\circ}$. On the other hand, a saturation phenomenon is presented after $270^{\circ}$ as shown in Figure 4, since after that point the capacity stabilizes and does not increase any further.

The major role of the scattering environment as well as the spatial correlation benefits can be envisaged also from the results in Figure 5 where the outage capacity results are presented as a function of the scatterer circle radius $S_{R}$. It is noteworthy to mention that the number of the scatterers $L$ is kept constant when the parameter $S_{R}$ is varied (we selected $a=2 \pi$ which corresponds to 100 scatterers). It is observed that as the radius decreases and the scatterers come closer to the antenna elements the overall capacity increases. Reducing the distance about to its half, the capacity increases each time approximately $1 \mathrm{~b} / \mathrm{s} / \mathrm{Hz}$ having either two or four elements at the receiver as shown in Figure 5. This can be attributed to the fact that since the number of the scatterers is constant and come closer to the antenna array, their path length angular separation becomes

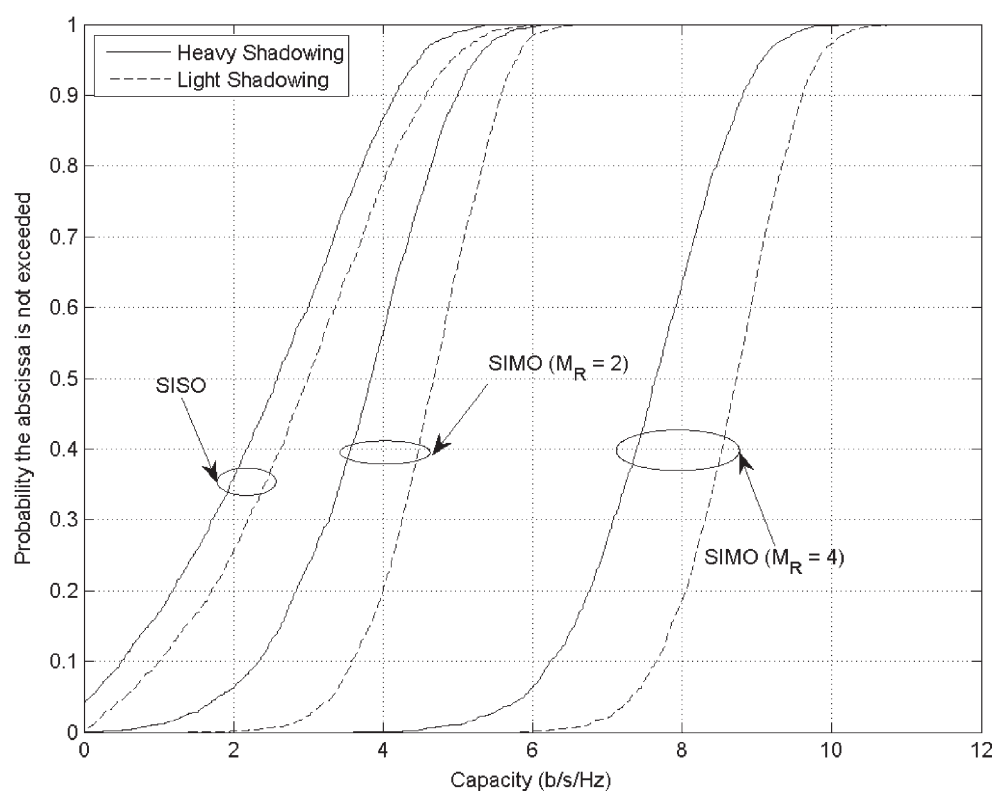

Figure 3 Capacity CDF for light and heavy shadowing scenario at C-band with two or four elements at the receiver terminal. Additionally, $\theta_{\text {elev }}=60^{\circ}, S_{R}=50 \mathrm{~m}$ and $a=2 \pi$. 


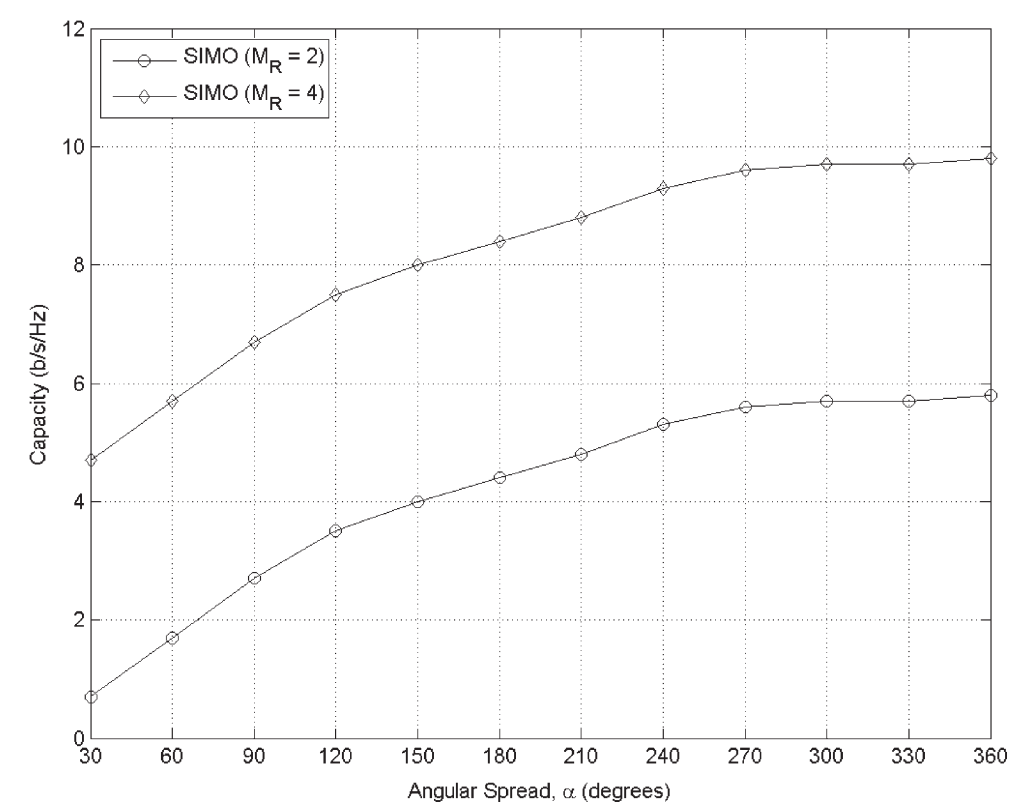

Figure 4 Average capacity as a function of the angular spread for a light shadowing scenario at C-band with two or four elements at the receiver terminal. Additionally, $\theta_{\text {elev }}=60^{\circ}$, and $S_{R}=50 \mathrm{~m}$.

larger. In advance, the correlation between these path lengths is reduced, improving the diversity gain and the channel capacity.

\section{Taking depolarization into account}

\section{Polarization effects and depolarization modelling}

Multipath propagation caused by scattering along the propagation path may cause random changes in the polarization state, which is also termed depolarization of the received field. This is a result of the electromagnetic investigation of wave propagation in a random, general scattering medium (see, e.g. [13], the classical work of propagation in random media). Similar results are also found in a rigorous investigation using full-wave electromagnetic simulation in a particular environment with well-specified scattering [14]. Experimental results are

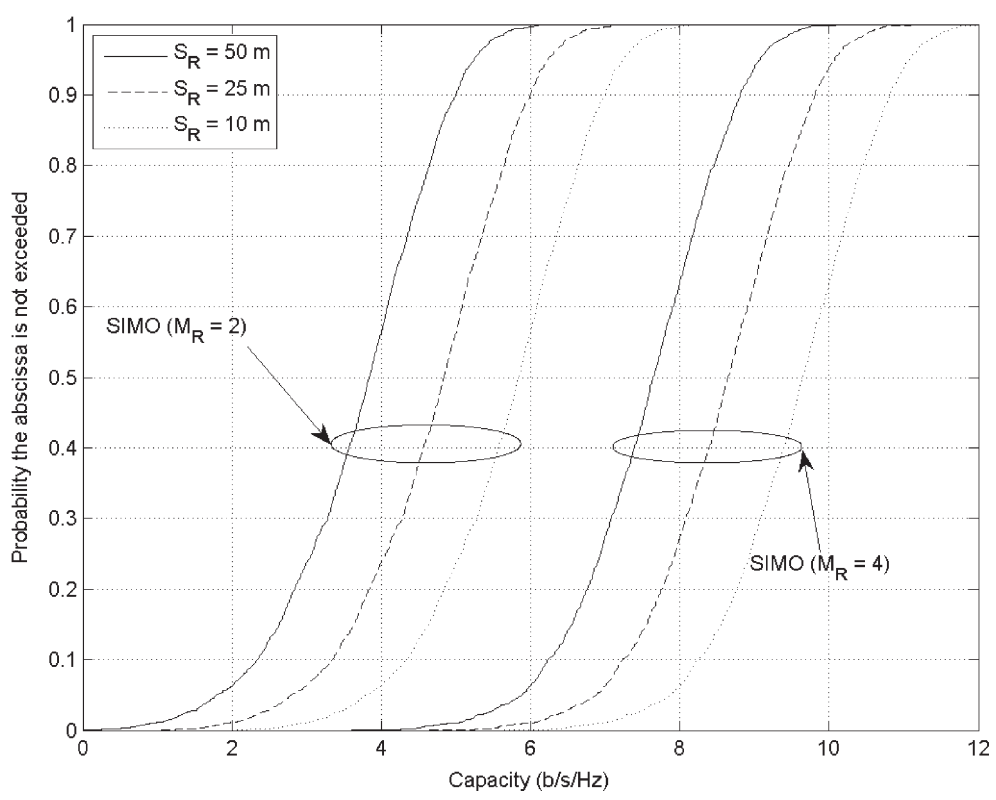

Figure 5 Capacity CDF as a function of the radius $S_{R}$ for a heavy shadowing scenario at C-band with two or four elements at the receiver terminal. The elevation angle is $60^{\circ}$ and $a=2 \pi$. 
also available. In [15], satellite-to-indoor propagation measurements are described; received field was measured with antennas of different polarization while transmitted field was elliptically polarized. Measured power was close to identical with receive antennas of co- and counter-rotating circular polarization and of arbitrary linear polarization. Nabar et al. [16] report on (terrestrial) measurements resulting in equal received power in the average of co-polar and cross-polar transmitted signals if the link length is $1.6 \mathrm{~km}$ or more. These and other results verify that the field at the receive antenna input port is depolarized. What is of interest is the power loss caused by polarization mismatch due to depolarization of the received field. For determining this loss, we have to take into account that (i) one particular realization of the received field at the antenna has one particular polarization being responsible for the possible polarization mismatch-loss; and (ii) this polarization is random.

The polarization state of an electromagnetic wave can be well described in the three-dimensional space of Stokes parameters. Stokes parameters and Stokes space were introduced by G. G. Stokes in the mid-1800 s as an appropriate mathematical tool for the description of polarization characteristics of electromagnetic radiation. It has been widely applied since then in optics; it is less frequently used in lower-than-optical frequency electromagnetic theory. The main advantage of applying these concepts here is the greater insight gained by using them. While polarization mismatch-loss can be determined by more direct methods, this insight, based on symmetry properties of Stokes space, leads to reasonable statistics. It would be difficult to arrive at these without this framework. A detailed description of the concept is given in [17]. Results of [17] applied in what follows are listed below. For the sake of simplicity it is assumed that polarization discrimination of the receive antenna is infinite, i.e. the transmission factor amounts to $T_{p}=0$ if the polarization of the incoming wave is orthogonal to that of the receive antenna.

In the general case, it is formally appropriate to describe this transmission factor as

$$
T_{p}=\frac{1}{2}\left(1+\sin \gamma_{i}\right)
$$

where $\gamma_{i}$ is a real angle characteristic of the "distance" between the antenna polarization state and that of the incoming wave. Polarization states of waves of equal power lay in the Stokes space on the surface of a sphere called the Poincaré sphere. This definition of Cartesian coordinates in the Stokes space is described amongst others in [17]. The relationship between spherical coordinates $(R, \gamma, \phi)$ of a point in Stokes space and the physical characteristics of the (polarized) electrical field $\left(E_{x}, E_{y}, \delta\right)$ are as follows $R=E_{x}^{2}+E_{y}^{2}, \gamma=\arcsin \left(\frac{2 E_{x} E_{y} \sin \delta}{E_{x}^{2}+E_{y}^{2}}\right)$, $\phi=\arctan \left(\frac{2 E_{x} E_{y} \cos \delta}{E_{x}^{2}-E_{y}^{2}}\right)$ with $E_{x}, E_{y}$ and $\delta$ being the $x$ and $y$ components of the electric field and the phase angle difference between these two, respectively, $\gamma$ is the elevation angle of any point in the Stokes space and $\phi$ is its azimuthal angle. In (7) (with some simplification), the elevation angle of the antenna polarization state is taken as $\pi / 2$ and $\gamma_{i}$ is the elevation angle of the incoming wave.

Various "fine structures" of polarization are possible in the case of random polarization; in one of these, all polarization states are equally likely. In this case, the probability distribution of the polarization state being at the neighbourhood of any special point of the sphere is uniform. On the other hand, less radical change of the polarization state is also likely. Thus, it is reasonable to assume that distribution is uniform over a solid angle $\Omega<4 \pi$. In this case, the probability density function of $T_{p}$ is given as

$$
p_{T_{p}}(x)= \begin{cases}\frac{2}{1-\sin \gamma_{0}}, & \text { if } \frac{1}{2}\left(1+\sin \gamma_{0}\right) \leq \mathrm{x} \leq 1 \\ 0, & \text { otherwise }\end{cases}
$$

If all polarization states are equally likely $\gamma_{0}=-\pi / 2$ and the distribution is uniform between 0 and 1 .

A similarly reasonable alternative assumption is that the polarization maintains its linear character but any orientation is equally likely. In that case

$$
p_{T_{p}}(x)=\frac{1}{\pi} \frac{1}{\sqrt{x(1-x)}}
$$

\section{Probability density of the received field-Rayleigh fading} In the case of depolarization the power received by the antenna is, due to polarization mismatch-loss, by a factor of $T_{p}$ lower than that at the antenna input port. In a fading channel, if the probability density function of the total power is $p_{\mathrm{P}}(x)$, the conditional density of the received power can be expressed as

$$
p_{\mathrm{P} \mid T_{p}}(x \mid y)=\frac{1}{y} p_{\mathrm{P}}\left(\frac{x}{y}\right)
$$

Assuming that any polarization is equally likely (i.e. $\left.\gamma_{0}=-\pi / 2\right)$ the total probability density of the power received by the antenna is

$$
p_{\mathrm{P}}(x)=\int_{0}^{1} p_{\mathrm{P} \mid T_{p}}(x \mid y) p_{T_{p}}(y) d y
$$


To be specific, assume Rayleigh fading. The received power is then exponentially distributed. Thus (11) leads to

$$
p_{\mathrm{P}}(x)=\frac{1}{M_{\mathrm{P}}} E_{1}\left(\frac{x}{M_{\mathrm{P}}}\right)
$$

with $M_{\mathrm{P}}$ being the expected value of the total power and $E_{1}(x)$ the exponential integral function, defined as $E_{1}(x) \equiv \int_{x}^{\infty} \frac{e^{-u}}{u} d u$. Notice that (12) fulfils the requirements of a probability density: it is non-negative and its integral is 1 . Furthermore, the average of the received power is $M_{\mathrm{P}} / 2$, i.e. the average loss due to depolarization is $3 \mathrm{~dB}$, as it should be. For the sake of completeness, we also give the more general formula for any value of $\gamma_{0}$ :

$$
\begin{aligned}
p_{P}(x)= & \frac{2}{M_{\mathrm{P}}\left(1-\sin \gamma_{0}\right)}\left[E_{1}\left(\frac{x}{M_{\mathrm{P}}}\right)\right. \\
& \left.-E_{1}\left(\frac{2 x}{M_{\mathrm{P}}\left(1+\sin \gamma_{0}\right)}\right)\right]
\end{aligned}
$$

Assuming, on the other hand, that depolarization is governed by the density of (9), similar calculation of distribution of received power gives

$$
p_{\mathrm{P}}(x)=\frac{1}{M_{\mathrm{P}}} \int_{0}^{1} \frac{e^{-x / y M_{\mathrm{P}}}}{y} \frac{1}{\pi} \frac{1}{\sqrt{y(1-y)}} d y=\frac{e^{-x / M_{\mathrm{P}}}}{\sqrt{\pi M_{\mathrm{P}} x}}
$$

The effect of depolarization on the channel capacity-the Rayleigh fading SISO case

It is well known that the capacity (in bits/channel use) of a Gaussian SISO channel can be expressed as

$$
C=\frac{\ln (1+\rho)}{\ln 2}
$$

In fading channels, the SNR is a random variable. Then the capacity is the expected value of (15), assuming that the channel is ergodic. Thus the (ergodic, i.e. average) capacity can be written as

$$
C_{\text {ergod }}=\frac{1}{\ln 2} \int_{0}^{\infty} \ln (1+f \rho) p_{f}(f) d f
$$

Here $\rho$ denotes the average SNR. In one realization, the actual SNR is $\rho$ multiplied by the random variable $f$; thus $f$ is normalized so that its expected value is 1 . Furthermore, $p_{f}(f)$ is the probability density function of the actual fading-Rayleigh, Rice, Corazza-Vatalaro or other fading models appropriate to satellite-to-earth links. In the present investigation, we first use (12) in place of $p_{f}$ $(f)$. Thus the expression of the ergodic capacity in the case of Rayleigh fading with $M_{\mathrm{P}}=1$ and complete depolarization is

$$
C_{\text {ergod }}=\frac{1}{\ln 2} \int_{0}^{\infty} \ln (1+f \rho) E_{1}(f) d f
$$

We know (based on Jensen's inequality) that in the case of high SNR for some specific capacity value the SNR difference between polarized and depolarized Rayleigh channels is more than $3 \mathrm{~dB}$ (while average loss due to depolarization is $3 \mathrm{~dB}^{\mathrm{a}}$ ). The result of (17) can be expressed in a closed form with the special general class of functions called Meijer's G-functions [18]. Accordingly, the ergodic capacity in the depolarized case is

$$
C_{\text {ergod }}=\frac{1}{\rho \ln 2} G_{2,3}^{3,1}\left(\frac{1}{\rho} \mid \begin{array}{l}
-1,1 \\
0,-1,-1
\end{array}\right)
$$

In Figure 6 we show the ergodic capacity versus the average SNR for Gaussian, Rayleigh and depolarized Rayleigh channels, respectively. The curves are presented for various $\gamma_{0}$ values, and were calculated by inserting (13) into (16). Results of Monte Carlo simulations are also overlaid on the figure, which confirm the analytical results. Focusing on the completely random depolarization $\left(\gamma_{0}=-\pi / 2\right.$ case $)$ again, it can be seen that in a SISO link the cost of depolarization in a Rayleigh fading channel (measured in terms of ergodic capacity) is about $4.3 \mathrm{~dB}$. This was further investigated by calculating the high-SNR power offset introduced in [19]. The high-SNR slope $\left(S_{\infty}\right)$ of the ergodic mutual information versus average SNR (in $\mathrm{dB}$ ) curve remains $S_{\infty}=1 \mathrm{bit} / \mathrm{s} /$ $\mathrm{Hz} /(3 \mathrm{~dB})$ as for pure Rayleigh fading, but it can be shown that the power offset $\left(L_{\infty}\right)$ expressed in $3 \mathrm{~dB}$ units becomes

$$
L_{\infty}=(1+\gamma) \log _{2} e
$$

Thus the SNR needs to be increased by $\log _{2} e \times$ $3 \mathrm{~dB} \approx 4.328 \mathrm{~dB}$ when compared to the pure Rayleigh case to obtain the same amount of mutual information, provided the SNR is high.Turning now to the case of completely though linearly depolarized Rayleigh channel based on (14) we have to insert the density

$$
p_{f}(f)=\frac{e^{-f}}{\sqrt{\pi f}}
$$

into (16). Capacity with depolarization can in this case also be given in closed form

$$
\begin{aligned}
C_{\mathrm{depol}}= & \frac{1}{\ln 2}\left[\pi \operatorname{Erfi}\left(\frac{1}{\sqrt{\rho}}\right)-\gamma-\ln \left(\frac{1}{\rho}\right)\right. \\
& \left.-\frac{1}{\rho}{ }_{2} F_{2}{ }^{2}(1,1 ; 3 / 2,2 ; 1 / \rho)\right]
\end{aligned}
$$




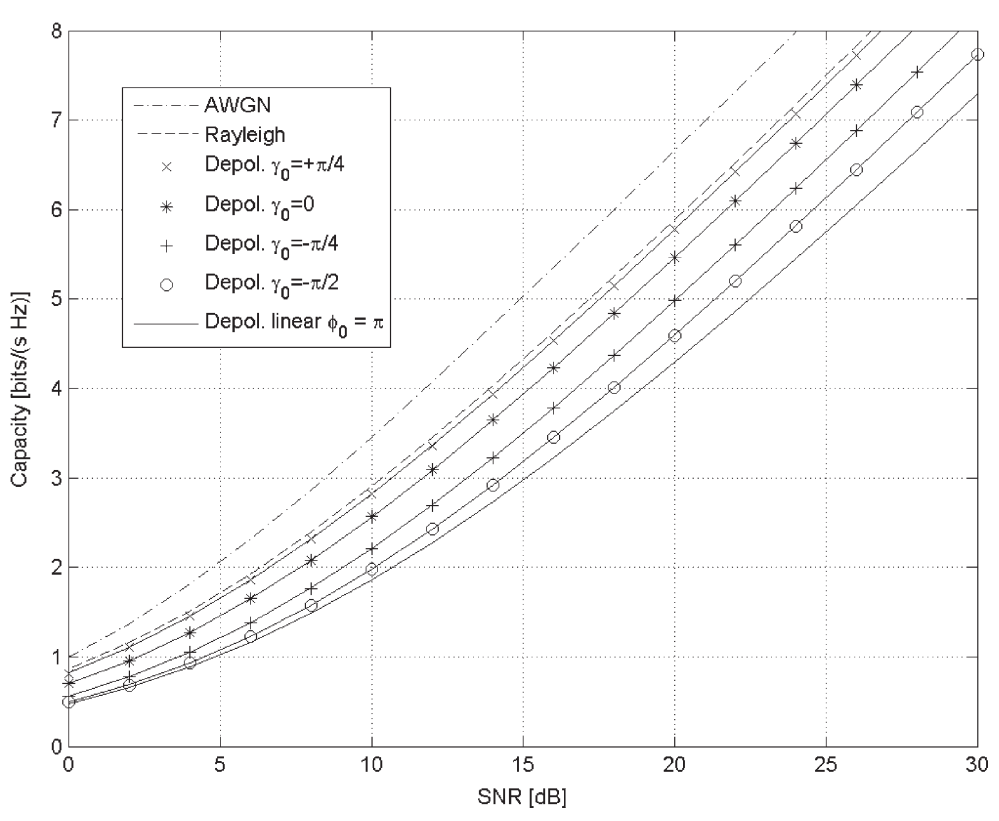

Figure 6 Average ergodic capacity of Rayleigh channels with complete and incomplete depolarization and with linear depolarization.

where $\operatorname{Erfi}(z)=j \operatorname{erf}(j z)$ is the imaginary error function and ${ }_{2} F_{2}(\cdot,,,,,, \cdot)$ is the hypergeometric function [20]. Results for the linear depolarization are also shown in Figure 6. As seen, the capacity is somewhat lower than that for arbitrary depolarization.

SIMO channel capacity-i.I.D. Depolarized Rayleigh fading Applying maximum ratio combining and assuming that antennas are placed so that received fields can be regarded as i.i.d, the probability density of received power is that of the convolution of component densities. Again that can be expressed in (a nearly) closed form for $M_{R}=2$ receive antennas (see [21], (2.5.11.6)):

$$
\begin{aligned}
p_{f, \mathrm{SIMO}}(a)= & \int_{0}^{a} E_{1}(x) E_{1}(a-x) d x \\
= & 2(\gamma+\ln a) e^{-a}+2(1-a \gamma-a \ln a) E_{1}(a) \\
& -a\left[\zeta(2)+(\gamma+\ln a)^{2}\right]-2 a \sum_{k=1}^{\infty} \frac{(-a)^{k}}{k ! k^{2}}
\end{aligned}
$$

where $\zeta(\cdot)$ is the Riemann zeta function. Inserting (22) into (16), we get the capacity of the depolarized SIMO channel in the presence of Rayleigh fading. The result is shown in Figure 7. Ergodic capacity was computed both via numerical integration of (22) and Monte Carlo simulation. For comparison, curves valid for related situations are also shown.

\section{SIMO channel capacity-taking depolarization into}

\section{account in situations of sections 2 and 3}

In the application of the above channel model, we assume that the specular ray of the received field is free of depolarization (i.e. $T_{p}=1$ ) while polarization of the scattered component is completely random (taking $\gamma_{0}=-\pi / 2-i . e$. the total Poincaré-sphere surface-in that case $T_{p}$ is uniformly distributed between 0 and 1 ). This seems to be realistic-based on simple physical insight as well as on measurement results of, e.g. [15]. Among the two depolarizing models described in Section 4.1, (7) is applied. Results are given in the sequel. Combining (4) and (7) the time-varying depolarized channel vector is obtained by

$$
\begin{aligned}
\tilde{\mathbf{h}}_{\mathrm{dep}}(t) & =\left[\begin{array}{lll}
\mathbf{a}\left(\theta_{R, 1}\right) & \ldots & \mathbf{a}\left(\theta_{R, L}\right)
\end{array}\right] \cdot \tilde{\mathbf{b}}\left(t, \theta_{R, i}\right) \\
& \cdot \sqrt{T_{p}\left(\theta_{R, i}\right)}
\end{aligned}
$$

Hence, if we combine (23) and (5) we can calculate the channel capacity considering a depolarization scenario. Figure 8 presents the capacity CDF at C-band, for a polarized and depolarized received field. The depolarization loss causes a capacity reduction of about $1.1 \mathrm{~b} / \mathrm{s} / \mathrm{Hz}$ (taking the median values from the CDF), having either two or four elements at the receiver antenna. Another finding, concerning SNR variability, is that, in case of a depolarized field, if we have four elements at the receiver and we require a capacity of $6.5 \mathrm{~b} / \mathrm{s} / \mathrm{Hz}$, the SNR have to be increased $4 \mathrm{~dB}$ so as to achieve the same capacity as in the polarized state.

Figure 9 presents the average capacity of a SIMO system at C-band as a function of angular spread for a 


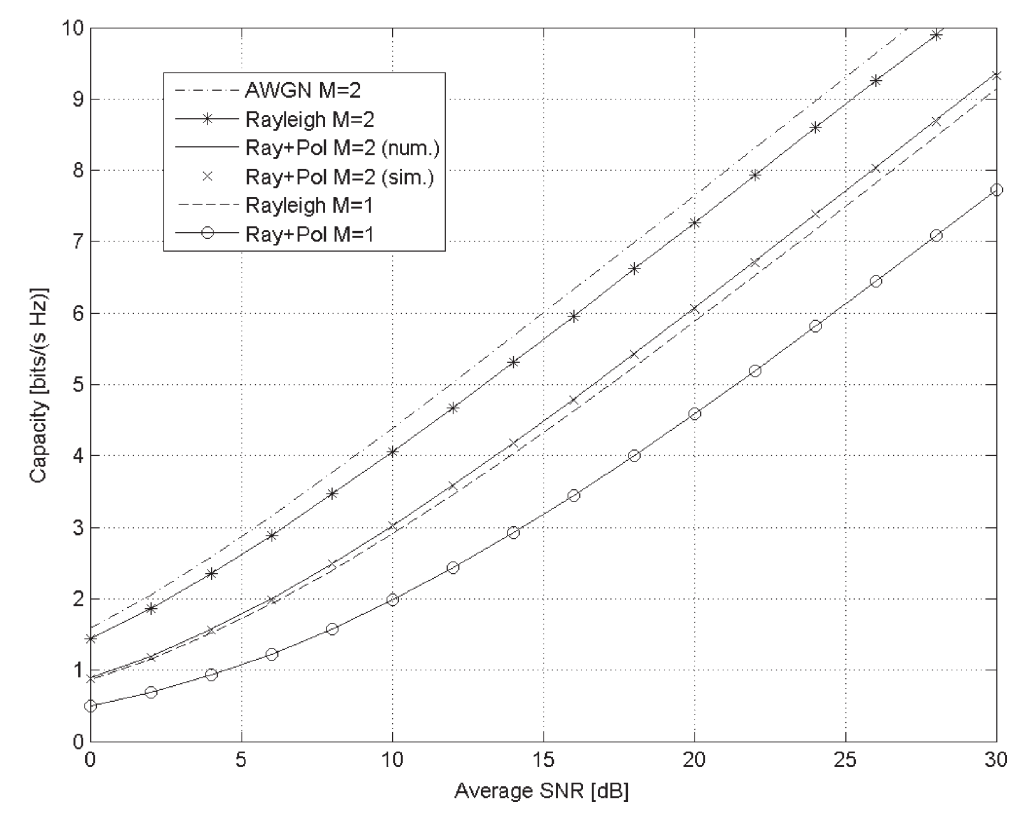

Figure 7 Ergodic capacity of various SISO and SIMO $\left(M_{R}=2\right)$ scenarios; Rayleigh fading; with MRC in the SIMO cases.

polarized and depolarized received field. Two or four elements are taken into account considering a light shadowing scenario. The angular spread takes on values between $30^{\circ}$ and $360^{\circ}$, in steps of $30^{\circ}$, and the average capacity is given by (6). In respect with Figure 4, the capacity increases as the sector $a$ increases since the multiple elements take advantage of the rich scattering environment. On the other hand, the introduced depolarization decreases further the diversity gain, causing in average a capacity drop of $0.7-0.9 \mathrm{~b} / \mathrm{s} / \mathrm{Hz}$ in comparison with the polarized state.

\section{Conclusions}

This study focused on the capacity evaluation of a satellite SIMO downlink at C-band considering either a light or heavy shadowed urban environment. Polarization

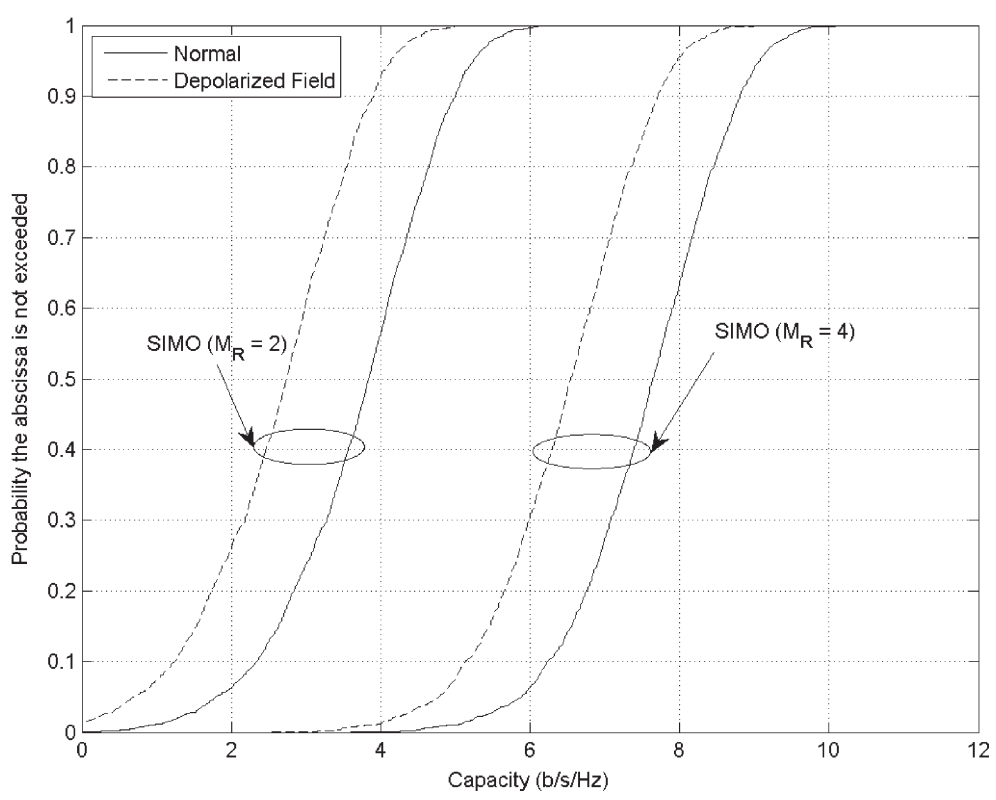

Figure 8 Capacity CDF taking depolarization into account at C-band with two or four elements at the receiver terminal. Heavy shadowing scenario is considered. Additionally, $\theta_{\text {elev }}=60^{\circ}, S_{R}=50 \mathrm{~m}$ and $a=2 \pi$. 


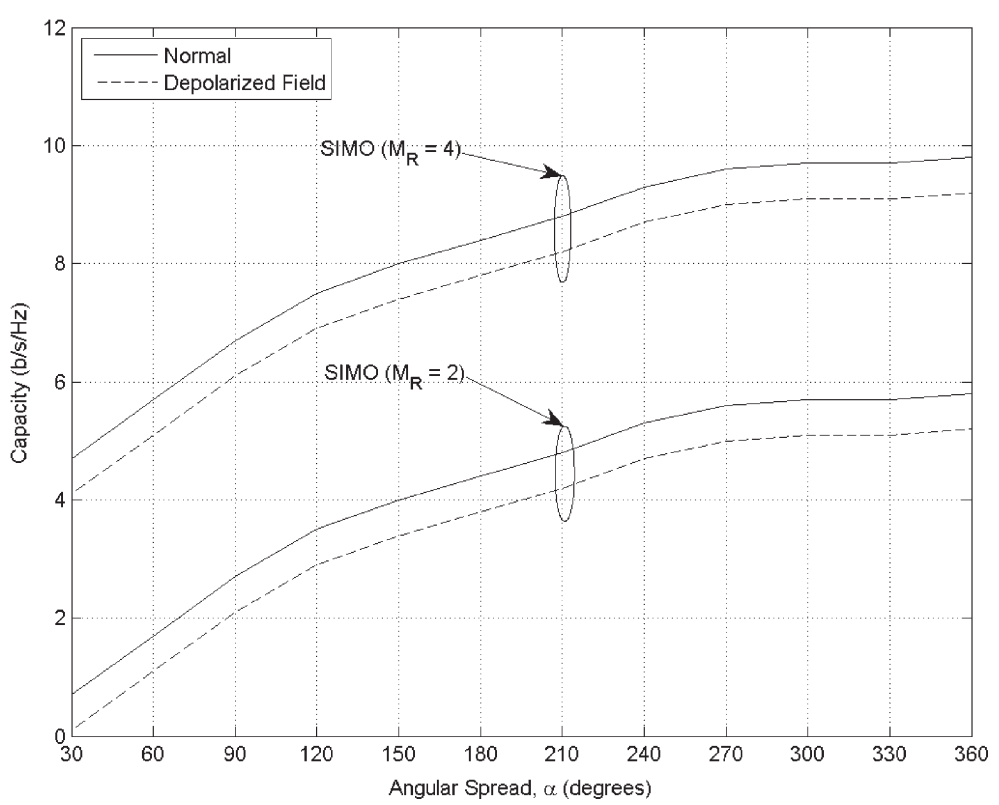

Figure 9 Average capacity as a function of angular spread taking depolarization into account at C-band with two or four elements at the receiver terminal. Light shadowing scenario is considered. Additionally, $\theta_{\text {elev }}=60^{\circ}, S_{R}=50 \mathrm{~m}$ and $a=2 \pi$.

effects are introduced and capacity results taking depolarization into account are presented. Overall, very sufficient capacities are achieved varying between 3.8 and $8.7 \mathrm{~b} / \mathrm{s} / \mathrm{Hz}$ in comparison with a SISO link. Additionally, in the heavy shadowing scenario the capacity drops significantly comparable with the light shadowing case. In average, the capacity decreases about $1 \mathrm{~b} / \mathrm{s} / \mathrm{Hz}$. The capacity increases as the angular spread increases since the multiple elements take advantage of the rich scattering environment and the lower spatial correlation.

The role of depolarization, causing additional loss, is usually neglected in non-dual-polarized situations. In this study it is taken into account, and in order to achieve this, a model of random received polarization based on Stokes parameters is given. Applying this model to Rayleigh channels, ergodic capacity in the case of depolarization is determined. If the received field is completely depolarized and every polarization is equally likely, an additional average power loss of $3 \mathrm{~dB}$ is formed (being a self evident result) whereas, in order to compensate that in ergodic capacity, an increase of about $4.1 \mathrm{~dB}$ is needed (the difference follows from Jensen's inequality). If the received field is completely depolarized and every linear polarization is equally likely, the difference is somewhat more (about $0.8 \mathrm{~dB}$ ). The capacity of a depolarized $1 \times 2$ SIMO diversity channel is nearly the same (by $0.5 \mathrm{~dB}$ higher) than that of a polarized SISO channel. In case of a SISO link the cost of depolarization considering a Rayleigh-fading channel is about $4.3 \mathrm{~dB}$. Finally, the average reduction of capacity due to depolarization in a SIMO link is approximately $1.1 \mathrm{bits} / \mathrm{s} / \mathrm{Hz}$.
If we have four elements at the receiver and a required capacity of $6.5 \mathrm{bits} / \mathrm{s} / \mathrm{Hz}$, in case of depolarization, we have to increase $4 \mathrm{~dB}$ the SNR so as to achieve the same capacity with the polarized case (note that this result is very close to the Rayleigh case).

\section{Endnote}

${ }^{a}$ As, by Jensen's inequality $\int_{0}^{\infty} \ln (1+f \rho) p_{f}(f) d f<$ $\ln \left(1+\rho \int_{0}^{\infty} f p_{f}(f) d f\right)$; or in words: expected value of a concave real function (e.g. $\ln x$ ) is less than the same function of the independent variable's expected value.

\section{Competing interests}

The authors declare that they have no competing interests.

\section{Acknowledgements}

This study was partially carried out in the framework of the European Network of Excellence IST-027393 SatNEx II, and partially in the framework of Thales "MIMOSA" project, Greek Ministry of Education.

\section{Author details}

'Institute of Communications and Computer Systems, National Technical University of Athens, Athens, Greece. ${ }^{2}$ Department of Broadband

Infocommunications and Electromagnetic Theory, Budapest University of Technology and Economics, Budapest, Hungary.

Received: 30 November 2011 Accepted: 14 May 2012

Published: 29 June 2012

\section{References}

1. P Driessen, G Foschini, On the capacity formula for multiple input-multiple output wireless channels: a geometric interpretation. IEEE Trans. Commun. 47(2), 173-176 (1999)

2. J Mietzner, P Hoeher, Distributed space-time codes for cooperative wireless networks in the presence of different propagation delays and path losses. in Proc. Sensor Array and Multichannel Signal Processing Workshop (Germany, 2004), pp. 264-pp. 268. July 2004 
3. PR King, BG Evans, S Stavrou, Physical-statistical model for the land mobilesatellite channel applied to satellite/HAP MIMO. in Proc. European Wireless Conference, (United Kingdom, April 2005), pp. 1-pp. 5

4. PR King, S Stavrou, Capacity improvement for a land mobile single satellite MIMO system. IEEE Antennas Wirel. Propagat. Lett. 5, 98-100 (2006)

5. PR King, S Stavrou, Low elevation wideband land mobile satellite MIMO channel characteristics. IEEE Trans. Wirel. Commun. 6(7), 2712-2720 (2007)

6. PR King, P Horváth, F Perez-Fontan, I Frigyes, S Stavrou, Satellite channel impairment mitigation by diversity techniques. in Proc. 14th IST Mobile and Wireless Communications Summit, (Dresden, Germany, June 2005)

7. P Horváth, GK Karagiannidis, PR King, S Stavrou, I Frigyes, Investigations in satellite MIMO channel modeling: accent on polarization. EURASIP J. Wirel. Commun. Netw. 2007, 10 (2007)

8. A Schmitz-Peiffer, L Stopfkuchen, J-J Floch, A Fernandez, R Jorgensen, B Eisfeller, JA Rodriguez, S Wallner, J-H Won, M Anghileri, B Lankl, T Schüler, O Balbach, E Colzi, Architecture for a future C-band/L-band GNSS mission. in InsideGNSS, (vol. May/June, 2009), pp. 47-pp. 56

9. I Frigyes, BG Molnár, R Vallet, Z Herczku, Z Bodnár, Doppler spread characteristics of satellite-personal communication channels. Int. J. Satell. Commun. 19, 251-262 (2001)

10. H Suzuki, A statistical model for urban radio propagation. IEEE Trans. Commun. 25(7), 673-680 (1977)

11. F Perez Fontan, A Mayo, D Marote, R Prieto Cerdeira, P Marino, F Machado, $N$ Riera Díaz, Review of generative models for the narrowband land mobile satellite propagation channel. Int. J. Satell. Commun. Netw. 26, 291-316 (2008)

12. A Paulraj, R Nabar, D Gore, Introduction to Space-Time Wireless Communications (Cambridge University Press, New York, 2003)

13. A Ishimaru, Wave Propagation and Scattering in Random Media (IEEE Press, Piscataway, NJ, 1997)

14. P Horváth, I Frigyes, Satellite-to-indoor propagation prediction using full-wave electromagnetic methods. in 4th Advanced Satellite Mobile Systems Conference (ASMS2008), (Bologna, Italy, 26-28 August 2008)

15. S Scalise, MA Díaz, J Bitó, M Bousquet, L Castanet, I Frigyes, P Horváth, A Jahn, M Krejcarek, J Lemorton, M Luglio, S Morosi, M Neri, M VázquezCastro, Digital Satellite Communications. Satellite Channel Impairments chapter, Springer, Berlin, 2007, pp. 65-pp. 115. ISBN 978-0-387-25634-4

16. R Nabar, H Bolcskei, V Erceg, D Gesbert, A Paulraj, Performance of multiantenna signaling techniques in the presence of polarization diversity. IEEE Trans. Signal Process 50(10), 2553-2562 (2002)

17. I Frigyes, BG Molnár, Z Bodnár, Z Herczku, Antenna gain and polarization effects in wireless links - accent on LEO satellites. Space Commun. 3-4, 199-208 (2005)

18. EW Weisstein, G-Function Meijer, MathWorld - a wolfram web resource, http://mathworld.wolfram.com/MeijerG-Function.html

19. A Lozano, A Tulino, S Verdu, High-SNR power offset in multiantenna communication. IEEE Trans Inf Theory 51(12), 4134-4151 (2005)

20. EW Weisstein, Hypergeometric Function. MathWorld - a wolfram web resource, http://mathworld.wolfram.com/HypergeometricFunction.html

21. AP Prudnikov, YA Brychkov, OI Marichev, Integraly i ryady, tom 2. Special'nye funkcii, Nauka, 1983

\section{Submit your manuscript to a SpringerOpen ${ }^{\circ}$ journal and benefit from:}

- Convenient online submission

- Rigorous peer review

- Immediate publication on acceptance

- Open access: articles freely available online

- High visibility within the field

- Retaining the copyright to your article

Submit your next manuscript at $\gg$ springeropen.com 